\title{
Production and decays of BSM Higgs bosons in various models
}

\section{Michael Spira*}

Paul Scherrer Institut, CH-5232 Villigen PSI, Switzerland

E-mail: michael.spira@psi.ch

\begin{abstract}
The recent discovery of the Higgs boson at the LHC marked the completion of the Standard Model (SM) of strong and electroweak interactions. I will summarize the theoretical ingredients involved in the production and decay processes at the LHC that allowed and will allow for a quite concise picture of the properties of the discovered resonance. These theoretical calculations are a crucial contribution to the discrimination of the SM from BSM scenarios at the LHC. I'll discuss the theoretical status of production cross sections and decay widths for MSSM Higgs bosons as an example of BSM Higgs bosons.
\end{abstract}

Prospects for Charged Higgs Discovery at Colliders - CHARGED2018

25-28 September 2018

Uppsala, Sweden

${ }^{*}$ Speaker. 


\section{Introduction}

The discovery of a Standard-Model-like Higgs boson at the LHC [1] completed the theory of electroweak and strong interactions. The measured Higgs mass of (125.09 \pm 0.24$) \mathrm{GeV}$ [2] ranges at the order of the weak scale. The existence of the Higgs boson [3] allows the SM particles to be weakly interacting up to high-energy scales. This, however, is only possible for particular Higgsboson couplings to all other particles so that with the knowledge of the Higgs-boson mass all its properties are uniquely fixed. The massive gauge bosons and fermions acquire mass through their interaction with the Higgs field that develops a finite vacuum expectation value in its ground state. The minimal model requires the introduction of one isospin doublet of the Higgs field and leads after spontaneous symmetry breaking to the existence of one scalar Higgs boson.

The minimal supersymmetric extension of the Standard Model (MSSM) introduces two Higgs doublets leading to five elementary Higgs bosons after electroweak symmetry breaking, two neutral CP-even (scalar) bosons $h, H$, one neutral CP-odd (pseudoscalar) boson $A$ and two charged bosons $H^{ \pm}$. The MSSM Higgs sector is determined by two input parameters at leading order, which are generally chosen as $\operatorname{tg} \beta=v_{2} / v_{1}$, the ratio of the two vacuum expectation values $v_{1,2}$, and the pseudoscalar Higgs mass $M_{A}$, if all SUSY parameters are real. Taking into account the oneloop and dominant two-loop corrections the upper bound on the light scalar Higgs mass is lifted to $M_{h} \lesssim 135 \mathrm{GeV}$ [4]. The additional three-loop results affect this upper bound by less than $1 \mathrm{GeV}$ [5]. The Higgs couplings to gauge bosons and fermions depend on the mixing angles $\alpha$ and $\beta$, which are fixed by diagonalizing the neutral and charged Higgs mass matrices. For large $\operatorname{tg} \beta$ values the down-type Yukawa couplings are enhanced and the up-type Yukawa couplings suppressed, if the light (heavy) scalar Higgs mass does not range at its upper (lower) bound, where the couplings become Standard-Model-like (up to a sign for the heavy scalar Higgs boson).

\section{Higgs Boson Decays}

For the SM, the determination of the branching ratios of Higgs-boson decays necessitates the inclusion of the available higher-order corrections [6] and a sophisticated estimate of the theoretical and parametric uncertainties. The parametric errors are dominated by the uncertainties in the top, bottom and charm quark masses as well as the strong coupling $\alpha_{s}$. We have used the $\overline{\mathrm{MS}}$ masses for the bottom and charm quark [7], $\bar{m}_{b}\left(\bar{m}_{b}\right)=(4.18 \pm 0.03) \mathrm{GeV}$ and $\bar{m}_{c}(3 \mathrm{GeV})=(0.986 \pm$ $0.026) \mathrm{GeV}$, and the top quark pole mass $m_{t}=(172.5 \pm 1) \mathrm{GeV}$ according to the conventions of the LHC Higgs Cross Section WG (HXSWG) [7]. The $\overline{\mathrm{MS}}$ bottom and charm masses are evolved from the input scale to the scale of the decay process with 4-loop accuracy in QCD. The strong coupling $\alpha_{s}$ is fixed by the input value at the $Z$-boson mass scale, $\alpha_{s}\left(M_{Z}\right)=0.118 \pm 0.0015$. The total parametric uncertainty for each branching ratio has been derived from a quadratic sum of the individual impacts of the input parameters on the decay modes.

The theoretical uncertainties within the SM from missing higher orders in the perturbative expansion are summarized in Table 1 for the individual partial decay processes along with the perturbative orders of the included QCD/elw. corrections [6]. In order to be conservative the total parametric uncertainties are added linearly to the theoretical uncertainties. For the SM, the final result for the branching ratios is shown in Fig. 1 for the leading Higgs decay modes with branching 


\begin{tabular}{lllll}
\hline Partial Width & QCD & Electroweak & Total & on-shell Higgs \\
\hline$H \rightarrow b \bar{b} / c \bar{c}$ & $\sim 0.2 \%$ & $\sim 0.5 \%$ & $\sim 0.5 \%$ & $\mathrm{~N}^{4} \mathrm{LO} / \mathrm{NLO}$ \\
$H \rightarrow \tau^{+} \tau^{-} / \mu^{+} \mu^{-}$ & - & $\sim 0.5 \%$ & $\sim 0.5 \%$ & $-/ \mathrm{NLO}$ \\
$H \rightarrow g g$ & $\sim 3 \%$ & $\sim 1 \%$ & $\sim 3 \%$ & $\mathrm{~N}^{3} \mathrm{LO} / \mathrm{NLO}$ \\
$H \rightarrow \gamma \gamma$ & $<1 \%$ & $<1 \%$ & $\sim 1 \%$ & $\mathrm{NLO} / \mathrm{NLO}$ \\
$H \rightarrow Z \gamma$ & $<1 \%$ & $\sim 5 \%$ & $\sim 5 \%$ & $\mathrm{LO} / \mathrm{LO}$ \\
$H \rightarrow W W / Z Z \rightarrow 4 f$ & $<0.5 \%$ & $\sim 0.5 \%$ & $\sim 0.5 \%$ & $\mathrm{NLO}$ \\
\hline
\end{tabular}

Table 1: Estimated theoretical uncertainties from missing higher orders and the perturbative orders (QCD/elw.) of the results included in the analysis.

ratio larger than $10^{-4}$ for the Higgs-mass range between 120 and $130 \mathrm{GeV}$. They have been obtained with Prophecy4F [8] for the decays $H \rightarrow W W, Z Z$ and HDECAY [9] for the other decay modes. The bands represent the total uncertainties of the individual branching ratios.

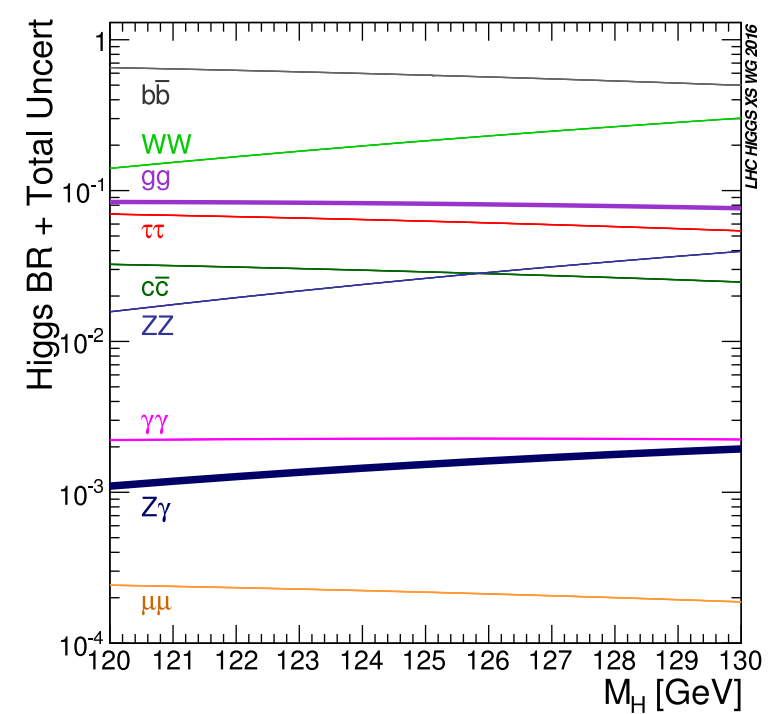

Figure 1: Higgs boson branching ratios and their uncertainties for Higgs masses around $125 \mathrm{GeV}$. From Ref. [7].

The three major differences between the decay widths of the SM Higgs boson and the MSSM Higgs bosons are the existence of 5 different Higgs bosons, the potentially possible and relevant decay modes into supersymmtric particles as squarks and gauginos and the numerically significant corrections to the bottom Yukawa coupling, the ' $\Delta_{b}$ ' corrections [10]. While the light scalar Higgs behaves as the SM Higgs boson in most viable MSSM scenarios, the decay profile of the heavy Higgs bosons $H, A, H^{ \pm}$is completely different. Examples of the MSSM Higgs branching ratios are shown in Fig. 2. A detailed discussion of the theoretical ingredients can be found e.g. in Ref. [6].

\section{Higgs Boson Production}

Within the SM the dominant Higgs production channel at the LHC is gluon fusion. While the NLO result is known exactly [11] the QCD corrections beyond NLO are known in the heavy-top- 

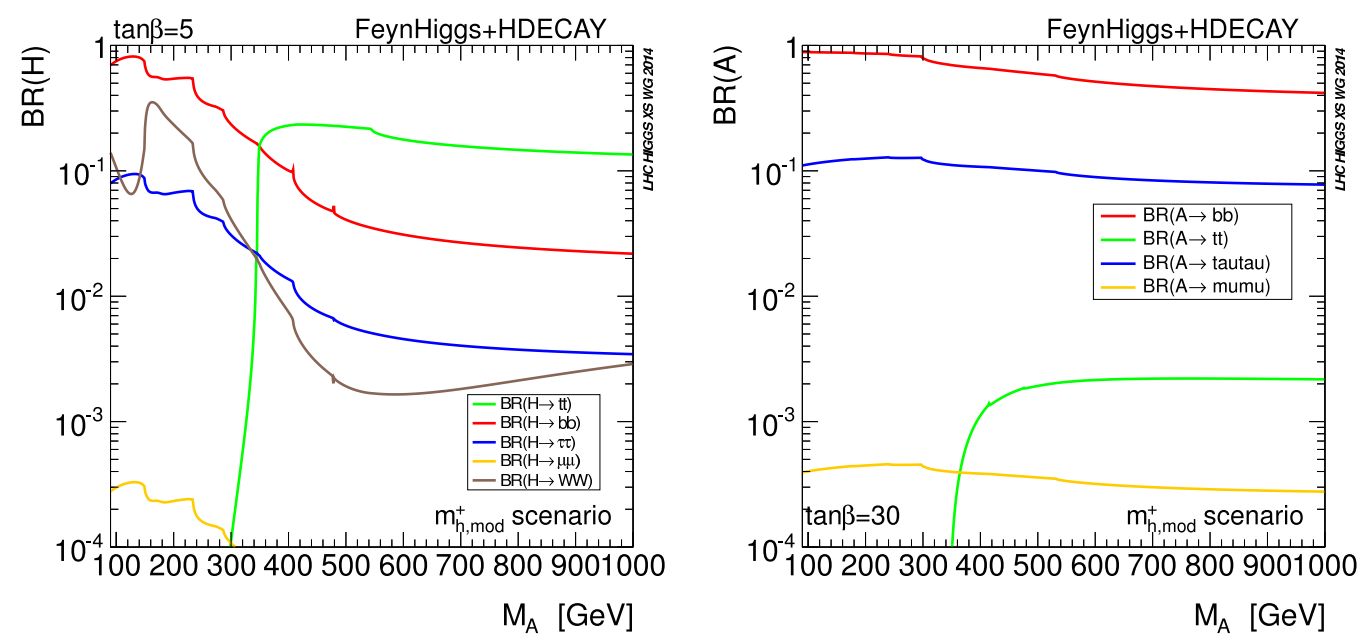

Figure 2: MSSM Higgs boson branching ratios for the heavy scalar and the pseudoscalar at two values of $\operatorname{tg} \beta$. From Ref. [7].

quark limit $[12,13,14]$ that, however, provides a reliable approximation for the inclusive cross section. Soft-gluon effects beyond $\mathrm{N}^{3} \mathrm{LO}$ turned out to be small [15]. The next sizable Higgs production mode is provided by vector-boson fusion. The QCD and elw. corrections are known at NLO exactly [16, 17], while QCD corrections are known up to $\mathrm{N}^{3} \mathrm{LO}$ within the structure-function approach [18]. Higgs-strahlung off $W / Z$ bosons is known at NLO elw. [19] and NNLO QCD [20]. Finally Higgs bremsstrahlung off top quarks is known at NLO QCD [21] and elw. [22], while single top plus Higgs boson production is known at NLO QCD [23].

The associated production of the Higgs boson with a $b \bar{b}$ pair is plagued by potentially sizable logarithms that emerge from the transverse-momentum integration of the final-state bottom quarks. The logarithms are related to the DGLAP evolution of bottom densities inside the proton. This leads to two different approaches for the calculation of this process, the 4-flavour scheme (4FS) and the 5-flavour scheme (5FS), where the first does not include bottom quarks in the proton and the running of $\alpha_{s}$, while the 5FS does. The advantage of the 4FS is that the full b-mass dependence and off-shell effects are kept at each perturbative order, while the 5FS requires massless and onshell bottom quarks, but resums the logarithms by the DGLAP evolution of the bottom densities of the proton. The typical factorization scale to be chosen for the bottom densities has been shown to be significantly smaller than the natural scale in the 5FS [24] so that the logarithms turn out to be of more moderate size. However, (up to finite bottom-mass effects) both approaches have to converge towards each other at higher orders. The 4FS calculation is known at NLO [25] and the 5FS calculation up to NNLO [26] in QCD. Both differ by about 20-30\%. Recently a consistent matching between both schemes has been performed in two different approaches [27] with mutual agreement within their respective uncertainties thus providing the best possible prediction with the present state of the art.

All Higgs boson production cross sections have been updated with the known higher-order corrections and the most recent parton density functions, i.e. the PDF4LHC15 sets [28], where NLO densities have been used consistently for NLO predictions and NNLO densities for NNLO predictions. Using the same values of the input parameters as for the branching ratios discussed before and their uncertainties a rigorous analysis has been performed to derive a reliable prediction 
of the central cross section values and their uncertainties. The results are shown in Fig. 3 as a function of the c.m. energy at the LHC for a Higgs mass $M_{H}=125 \mathrm{GeV}$. The size of the coloured bands represents the individual sums of the theoretical and parametric uncertainties. All production cross sections with results beyond NLO in QCD exhibit a small residual uncertainty in the few-per-cent range. Only the cross sections for $t \bar{t} H, b \bar{b} H$ and $t H$ production develop larger uncertainties. The theoretical and parametric uncertainties of each production process have been added in quadrature. The gluon-fusion cross sections can be predicted with a total (Gaussian) uncertainty of about 5\%, the vector-boson-fusion and $W H$ Higgs-strahlung channels with less than $3 \%$ uncertainty and the ZH Higgs-strahlung channel with about $4 \%$ uncertainty due to the novel loop contributions from $g g \rightarrow Z H$. The two-loop QCD corrections to the latter process are known in the limit of heavy top quarks [29]. The uncertainties of $t \bar{t} H$ production amount to about $10-15 \%$, for $s$ - and $t$-channel $t H$ production to about $15-20 \%$ and for $b \bar{b} H$ production to about $20-25 \%$.

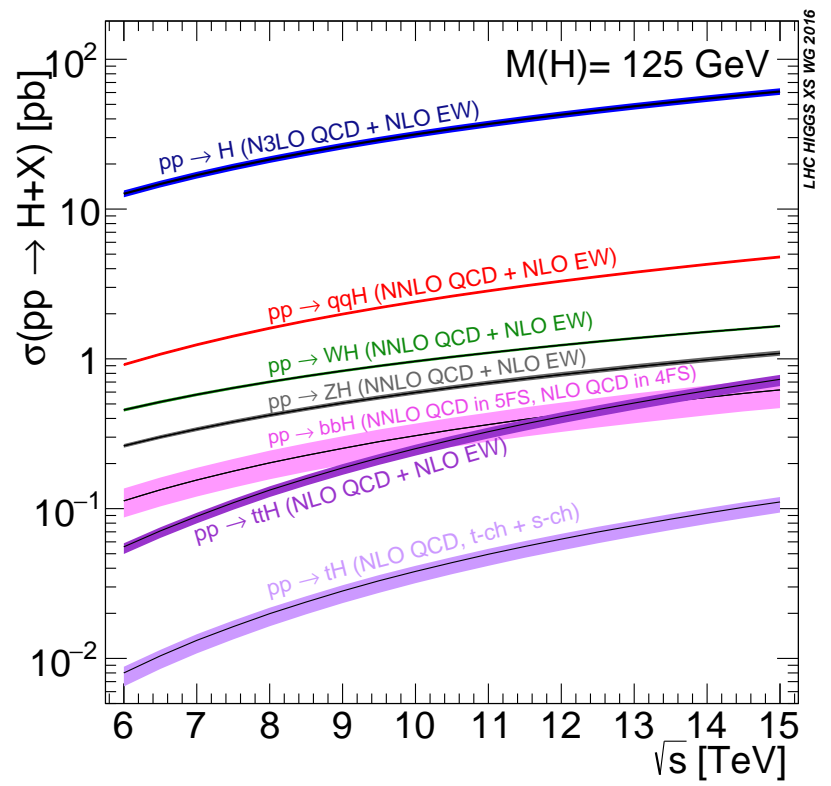

Figure 3: Higgs boson production cross sections as a function of the c.m. energy at the LHC for a Higgs mass $M_{H}=125 \mathrm{GeV}$ including the most up-to-date higher-order corrections as indicated at the shown cross section bands. The size of the bands reflects the total estimated theoretical uncertainties. From Ref. [7].

In the MSSM there are additional contributions of squarks to the gluon-fusion mechanism that are sizeable for squark masses below about $500 \mathrm{GeV}$. The pure QCD corrections [30] and the full SUSY-QCD corrections [31] have been calculated at NLO resulting in a similar large increase of the cross sections for the QCD part, while the sign of the SUSY-QCD part depends mainly on the sign of the $\mu$ parameter. On the other hand the genuine SUSY-QCD and SUSY-elw. corrections to vector-boson fusion and Higgs-strahlung are small [32], while the SUSY-QCD corrections to the assciated MSSM Higgs production with a $t \bar{t}$ pair are of moderate size [33]. For $b \bar{b}+H / A$ production the genuine SUSY-QCD/elw. corrections can be reliably approximated by the $\Delta_{b}$ terms [33]. The dominant charged Higgs production mode is the associated production with a top and bottom quark. The QCD corrections are sizeable [34, 35], while the genuine SUSY-QCD part is of moderate size for small $\operatorname{tg} \beta$, and for $\operatorname{large} \operatorname{tg} \beta$ it can be reliably approximated by the $\Delta_{b}$ corrections [34]. 
Higgs-boson pair production will allow for the first time to probe the trilinear Higgs selfcoupling directly and thus the first part of the Higgs potential as the origin of electroweak symmetry breaking. The dominant Higgs pair production mode is gluon fusion $g g \rightarrow H H$ that is loop-induced at LO and mediated by top and to a much lesser extent bottom loops [36]. The total gluon-fusion cross section is about three orders of magnitude smaller than the corresponding single-Higgs production cross section. The dependence of the gluon-fusion cross section on the trilinear Higgs coupling $\lambda$ is approximately given by $\Delta \sigma / \sigma \sim-\Delta \lambda / \lambda$ so that the uncertainties of the cross section are immediately translated into the uncertainty of the extracted trilinear coupling $\lambda$. In order to reduce the uncertainties of the cross section higher-order corrections are required. The NLO QCD corrections have first been obtained in the heavy-top limit [37] supplemented by a large topmass expansion [38] and the inclusion of the full real corrections [39]. Quite recently the full NLO calculation including the full top-mass dependence has been performed [40, 41, 42] showing a 15\%-difference to the result obtained in the heavy-top limit for the total cross section. For the distributions the differences can reach $20-30 \%$ for large invariant Higgs pair masses. The full NLO results are shown in Fig. 4. The NLO QCD corrections increase the cross section by close to
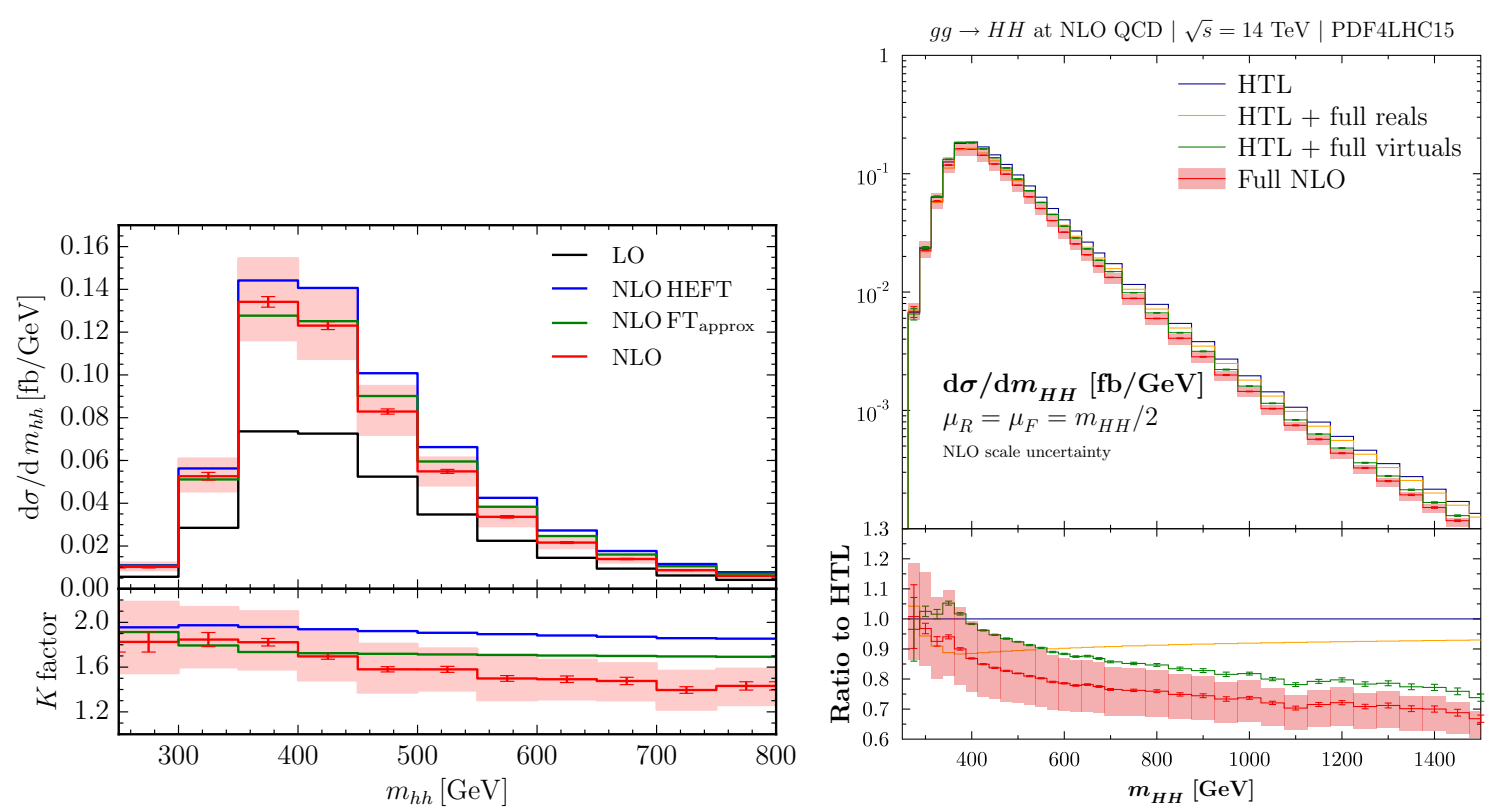

Figure 4: Higgs boson pair production cross sections as a function of the invariant Higgs pair mass at the LHC for a Higgs mass $M_{H}=125 \mathrm{GeV}$ including the NLO QCD corrections. The size of the bands reflects the total renormalization and factorization scale uncertainties. From (left) Ref. [40] and (right) Ref. [42].

a factor two. Within the heavy top limit the NNLO QCD corrections have been derived and raise the cross section by a moderate amount of $20-30 \%$ thus signalizing perturbative convergence in analogy to the single-Higgs case [43]. The soft-gluon resummation adds only less than about $10 \%$ beyond NNLO [44]. Recently the full NLO result and the NNLO corrections in the heavy-top limit have been combined in a fully exclusive Monte Carlo program [45] that is publicly available. Moreover, the matching of the full NLO results to parton showers has been performed [46] so that there are complete NLO event generators containing these corrections.

The subleading Higgs pair production cross sections consist of vector-boson fusion $q q \rightarrow$ $q q H H$ that is known up to $\mathrm{N}^{3} \mathrm{LO}$ QCD $[47,48]$ in the structure-function approach, the associated 
Higgs pair production with a top pair $g g, q \bar{q} \rightarrow t \bar{t} H H$ that is known up to NLO QCD [39] and double Higgs-strahlung $q \bar{q} \rightarrow V H H(V=W, Z)$ that is known up to NNLO QCD [47]. The different production cross sections are shown in Fig. 5 as a function of the hadronic c.m. energy.

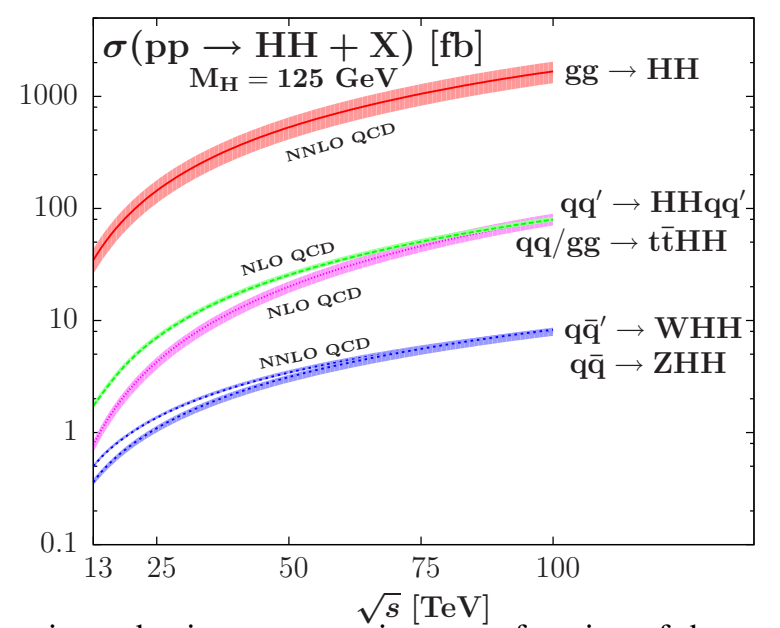

Figure 5: Higgs boson pair production cross sections as a function of the c.m. energy at hadron coliders for a Higgs mass $M_{H}=125 \mathrm{GeV}$ including the most up-to-date higher-order corrections as indicated at the shown cross section bands. The size of the bands reflects the total estimated theoretical uncertainties. From Ref. [49].

\section{References}

[1] G. Aad et al. [ATLAS Coll.], Phys. Lett. B716 (2012) 1; S. Chatrchyan et al. [CMS Coll.], Phys. Lett. B716 (2012) 30.

[2] G. Aad et al. [ATLAS and CMS Collaborations], JHEP 1608 (2016) 045.

[3] P. W. Higgs, Phys. Lett. 12 (1964) 132, Phys. Rev. Lett. 13 (1964) 508 and Phys. Rev. 145 (1966) 1156; F. Englert and R. Brout, Phys. Rev. Lett. 13 (1964) 321; G. S. Guralnik, C. R. Hagen and T. W. Kibble, Phys. Rev. Lett. 13 (1964) 585; T. W. B. Kibble, Phys. Rev. 155 (1967) 1554.

[4] See e.g. G. Degrassi et al., Eur. Phys. J. C28 (2003) 133.

[5] S. P. Martin, Phys. Rev. D75 (2007) 055005; R. V. Harlander et al., Phys. Rev. Lett. 100 (2008) 191602, Erratum ibid. 101 (2008) 039901; R. V. Harlander et al., Eur. Phys. J. C77 (2017) no.12, 814.

[6] M. Spira, Prog. Part. Nucl. Phys. 95 (2017) 98 and references therein.

[7] D. de Florian et al. [LHC Higgs Cross Section Working Group], arXiv:1610.07922 [hep-ph].

[8] A. Bredenstein et al., Phys. Rev. D74 (2006) 013004 and JHEP 0702 (2007) 080.

[9] A. Djouadi et al., Comput. Phys. Commun. 108 (1998) 56; A. Djouadi et al., arXiv:1801.09506 [hep-ph].

[10] L. J. Hall et al., Phys. Rev. D50, 7048 (1994); R. Hempfling, Phys. Rev. D49, 6168 (1994); M. Carena et al., Nucl. Phys. B426 (1994) 269; D. M. Pierce et al., Nucl. Phys. B491, 3 (1997); J. Guasch et al., Phys. Lett. B515 (2001) 367; G. D’ Ambrosio et al., Nucl. Phys. B645, 155 (2002); A. J. Buras et al., Nucl. Phys. B659, 3 (2003); V. Barger et al., Phys. Rev. D79, 115018 (2009); . D. Christensen et al., Phys. Rev. D85, 115018 (2012); M. S. Carena et al., Nucl. Phys. B577, 88 (2000); J. Guasch et al., 
Phys. Rev. D68, 115001 (2003). D. Noth and M. Spira, Phys. Rev. Lett. 101, 181801 (2008) and JHEP 1106, 084 (2011); L. Mihaila and C. Reisser, JHEP 1008, 021 (2010); A. Crivellin and C. Greub, Phys. Rev. D87 (2013) 015013 Erratum: [Phys. Rev. D87 (2013) 079901].

[11] A. Djouadi et al., Phys. Lett. B264 (1991) 440; S. Dawson, Nucl. Phys. B359 (1991) 283;

D. Graudenz et al., Phys. Rev. Lett. 70 (1993) 1372; M. Spira et al., Nucl. Phys. B453 (1995) 17;

R. Harlander and P. Kant, JHEP 0512 (2005) 015; S. Actis et al., Phys. Lett. B670 (2008) 12 and Nucl. Phys. B811 (2009) 182. C. Anastasiou et al., JHEP 0910 (2009) 068;

[12] S. Catani et al., JHEP 0105 (2001) 025; R. V. Harlander and W. B. Kilgore, Phys. Rev. D64 (2001) 013015 and Phys. Rev. Lett. 88 (2002) 201801; C. Anastasiou and K. Melnikov, Nucl. Phys. B646 (2002) 220; V. Ravindran et al., Nucl. Phys. B665 (2003) 325; S. Marzani et al., Nucl. Phys. B800 (2008) 127.

[13] T. Gehrmann et al., JHEP 1202 (2012) 056; C. Anastasiou et al., JHEP 1307 (2013) 003;

C. Anastasiou et al., JHEP 1312 (2013) 088; W. B. Kilgore, Phys. Rev. D89 (2014) 7, 073008; Y. Li et al., Phys. Rev. D90 (2014) 5, 053006; C. Anastasiou et al., JHEP 1503 (2015) 091; C. Anastasiou et al., JHEP 1605 (2016) 058; B. Mistlberger, JHEP 1805 (2018) 028.

[14] C. Anastasiou et al., Phys. Rev. Lett. 114 (2015) 212001.

[15] D. de Florian et al., JHEP 1410 (2014) 176; M. Bonvini and L. Rottoli, Phys. Rev. D91 (2015) 5, 051301. S. Catani et al., Nucl. Phys. B888 (2014) 75; D. de Florian and M. Grazzini, Phys. Lett. B718 (2012) 117; M. Bonvini and S. Marzani, JHEP 1409 (2014) 007; T. Schmidt and M. Spira, Phys. Rev. D93 (2016) no.1, 014022; M. Bonvini et al., JHEP 1608 (2016) 105.

[16] T. Han et al., Phys. Rev. Lett. 69 (1992) 3274; T. Figy et al., Phys. Rev. D68 (2003) 073005; T. Figy and D. Zeppenfeld, Phys. Lett. B591 (2004) 297; E. L. Berger and J. M. Campbell, Phys. Rev. D70 (2004) 073011.

[17] M. Ciccolini et al., Phys. Rev. Lett. 99 (2007) 161803 and Phys. Rev. D77 (2008) 013002;

[18] P. Bolzoni et al., Phys. Rev. Lett. 105 (2010) 011801 and Phys. Rev. D85 (2012) 035002; M. Cacciari et al., Phys. Rev. Lett. 115 (2015) no.8, 082002; F. A. Dreyer and A. Karlberg, Phys. Rev. Lett. 117 (2016) no.7, 072001.

[19] M. L. Ciccolini et al., Phys. Rev. D68 (2003) 073003; A. Denner et al., JHEP 1203 (2012) 075.

[20] T. Han and S. Willenbrock, Phys. Lett. B273 (1991) 167; O. Brein et al., Phys. Lett. B579 (2004) 149; G. Ferrera et al., JHEP 1404 (2014) 039 and Phys. Lett. B740 (2015) 51.

[21] W. Beenakker et al., Phys. Rev. Lett. 87 (2001) 201805 and Nucl. Phys. B653 (2003) 151; L. Reina and S. Dawson, Phys. Rev. Lett. 87 (2001) 201804; S. Dawson et al., Phys. Rev. D67 (2003) 071503;

[22] Y. Zhang et al., Phys. Lett. B 738 (2014) 1; S. Frixione et al., JHEP 1409 (2014) 065 and JHEP 1506 (2015) 184.

[23] F. Demartin et al., Eur. Phys. J. C75 (2015) no.6, 267.

[24] D. L. Rainwater et al., hep-ph/0203187; M. Spira, hep-ph/0211145; F. Maltoni et al., Phys. Rev. D67 (2003) 093005; E. Boos and T. Plehn, Phys. Rev. D69 (2004) 094005; J. M. Campbell et al., hep-ph/0405302; F. Maltoni et al., JHEP 1207 (2012) 022 Erratum: [JHEP 1304 (2013) 095]; M. Lim et al., JHEP 1609 (2016) 132.

[25] S. Dittmaier et al., Phys. Rev. D70 (2004) 074010; S. Dawson et al., Phys. Rev. D69 (2004) 074027; M. Wiesemann et al., JHEP 1502 (2015) 132. 
[26] R. V. Harlander and W. B. Kilgore, Phys. Rev. D68 (2003) 013001.

[27] S. Forte et al., Phys. Lett. B751 (2015) 331 and Phys. Lett. B763 (2016) 190; M. Bonvini et al., JHEP 1511 (2015) 196 and JHEP 1610 (2016) 053.

[28] J. Butterworth et al., J. Phys. G43 (2016) 023001.

[29] L. Altenkamp et al., JHEP 1302 (2013) 078; A. Hasselhuhn et al., arXiv:1611.05881 [hep-ph].

[30] S. Dawson et al., Phys. Rev. Lett. 77 (1996) 16; M. Mühlleitner and M. Spira, Nucl. Phys. B790 (2008) 1; R. Bonciani et al., JHEP 0711 (2007) 095.

[31] R. V. Harlander and M. Steinhauser, Phys. Lett. B574 (2003) 258 and JHEP 0409 (2004) 066; G. Degrassi and P. Slavich, Nucl. Phys. $B 805$ (2008) 267; G. Degrassi et al., Eur. Phys. J. C72 (2012) 2032; R. V. Harlander and F. Hofmann, JHEP 0603 (2006) 050; R. V. Harlander et al., JHEP 1102 (2011) 055; G. Degrassi et al., JHEP 1108 (2011) 128; G. Degrassi and P. Slavich, JHEP 1011 (2010) 044; C. Anastasiou et al., Phys. Rev. Lett. 100 (2008) 241806; M. Mühlleitner et al., PoS RADCOR 2009 (2010) 043 and DESY-PROC-2010-01.

[32] A. Djouadi and M. Spira, Phys. Rev. D62 (2000) 014004; W. Hollik et al., Phys. Rev. Lett. 102 (2009) 091802; T. Figy et al., JHEP 1202 (2012) 105.

[33] S. Dittmaier et al., JHEP 0703 (2007) 114. S. Dawson et al., Phys. Rev. D83 (2011) 115007; P. Wu et al., Phys. Lett. B618 (2005) 209; S. Dittmaier et al., Phys. Rev. D90 (2014) no.3, 035010.

[34] S. Dittmaier et al., Phys. Rev. D83 (2011) 055005; G. P. Gao et al., Phys. Rev. D66 (2002) 015007; S. H. Zhu, Phys. Rev. D67 (2003) 075006; T. Plehn, Phys. Rev. D67 (2003) 014018; E. L. Berger et al., Phys. Rev. D71 (2005) 115012; N. Kidonakis, PoS HEP 2005 (2006) 336.

[35] C. Degrande et al., Phys. Lett. B772 (2017) 87.

[36] E. W. N. Glover and J. J. van der Bij, Nucl. Phys. B309 (1988) 282; T. Plehn et al., Nucl. Phys. B479 (1996) 46 Erratum: [Nucl. Phys. B531 (1998) 655].

[37] S. Dawson et al., Phys. Rev. D58 (1998) 115012.

[38] J. Grigo et al., Nucl. Phys. B875 (2013) 1; J. Grigo et al., Nucl. Phys. B900 (2015) 412.

[39] R. Frederix et al., Phys. Lett. B732 (2014) 142; F. Maltoni et al., JHEP 1411 (2014) 079.

[40] S. Borowka et al., Phys. Rev. Lett. 117 (2016) no.1, 012001 Erratum: [Phys. Rev. Lett. 117 (2016) no.7, 079901].

[41] S. Borowka et al., JHEP 1610 (2016) 107.

[42] J. Baglio et al., arXiv:1811.05692 [hep-ph].

[43] D. de Florian and J. Mazzitelli, Phys. Lett. B724 (2013) 306 and Phys. Rev. Lett. 111 (2013) 201801; J. Grigo et al., Nucl. Phys. B888 (2014) 17.

[44] D. Y. Shao et al., JHEP 1307 (2013) 169; D. de Florian and J. Mazzitelli, JHEP 1509 (2015) 053.

[45] D. de Florian et al., JHEP 1609 (2016) 151.

[46] G. Heinrich et al., JHEP 1708 (2017) 088.

[47] J. Baglio et al., JHEP 1304 (2013) 151.

[48] L. S. Ling et al., Phys. Rev. D89 (2014) no.7, 073001; F. A. Dreyer and A. Karlberg, arXiv:1811.07918 [hep-ph] and arXiv:1811.07906 [hep-ph].

[49] J. Baglio et al., Rept. Prog. Phys. 79 (2016) no.11, 116201. 